\title{
Flavor-changing neutral current in production and decay of pseudoscalar mesons in a type III two-Higgs-doublet-model with four-texture Yukawa couplings
}

\author{
M. Gómez-Bock ${ }^{1}$, G. López-Castro ${ }^{2}$, L. López-Lozano ${ }^{3}$, A. Rosado ${ }^{3}$ \\ ${ }^{1}$ Instituto de Física, Universidad Nacional Autónoma de México, \\ Apartado Postal 20-364, México 01000 D.F., México \\ ${ }^{2}$ Departamento de Física, Cinvestav, \\ Apartado Postal 14-740, México 07000 D.F., México. \\ ${ }^{3}$ Instituto de Física, Benemérita Universidad Autónoma de Puebla. \\ Apartado Postal J-48, C.P. 72570 Puebla, Pue., México.
}

(Dated: October 30, 2018)

\begin{abstract}
We study flavor violating processes in the production or decay of a neutral pseudoscalar meson $P^{0}$ in the framework of a type III two Higgs Doublet Model with four-texture Yukawa couplings. We use a version of the model where Yukawa interactions of neutral Higgs bosons allow for flavor change at the tree-level, but conserves CP symmetry. The physical Yukawa couplings respect $\mathrm{CP}$-invariance due to the Hermiticity conditions that we impose on the fermion mass matrices. We focus on all possible $\tau^{ \pm} \rightarrow l^{ \pm} P^{0}$ and $P^{0} \rightarrow l^{+} l^{\prime-}$ decay channels, where $l, l^{\prime}$ are charged leptons. We find that these processes provide complementary information on quark and lepton FCNC Yukawa couplings. In particular flavor violating parameters in the quark sector, $\chi_{s b}$ and $\chi_{d b}$, are significantly constrained by present experimental data, whereas the corresponding parameters in the leptonic sector are less constrained.

PACS numbers: 12.60.Fr, 13.20.-v, 13.35.Dx, 14.80.Cp
\end{abstract}

\section{INTRODUCTION}

Flavor-changing neutral current (FCNC) processes and charge-parity (CP) symmetry violation observed in the quark sector are known to be duly explained in the standard model (SM) framework via the quark mixing mechanism [1]. Nowadays, experimental searches of CP violation and FCNC's in the leptonic sector are among the most interesting problems in particle physics since they would eventually shed some light on the origin of mixing and masses of leptons. Actually, since LF violating processes originating from mixing of neutrinos are expected to occur at an unobservable small level [2, 3], their observation would clearly indicate evidence for New Physics beyond the SM [4]. 
In this paper, we study the LF violating processes that may occur in the production or decay of a neutral pseudoscalar meson $P^{0}$ due to the exchange of a neutral pseudoscalar Higgs boson $\left(A^{0}\right)$. To be more specific, we will focus on LF violation in all possible $\tau^{ \pm} \rightarrow l^{ \pm} P^{0}$ and $P^{0} \rightarrow l^{+} l^{\prime-}$ decay channels $\left(l, l^{\prime}\right.$ are charged leptons) that are allowed by kinematics. Although our main focus is on LF violating processes, for the purposes of comparison we will also consider LF conserving decays of neutral heavy mesons by assuming that they are induced by the Higgs boson $A^{0}$ alone.

With the advent of $\mathrm{B}$ meson factories, large sets of $\tau$ lepton pairs have been accumulated by both BaBar and Belle experiments [5] allowing to improve previous bounds on flavor violating $\tau$ and $B$ meson decays. We note also that the flavor violating decays of our interest have been studied in a large variety of New Physics models and using different approximations [6, 7]. A comprehensive set of bounds on R-parity violating couplings from these LFV decays were obtained in [8]. In this paper we study these decays in the framework of a general two Higgs doublet model type-III (2HDM-III) [9] which contains flavor violation via Yukawa interactions of the neutral Higgs bosons at the tree-level. In this paper we work in a type III $2 \mathrm{HDM}$ with four-texture Yukawa couplings, where the Yukawa matrices are assumed to be Hermitean, this implies that CP symmetry is respected by the Yukawa interactions of neutral Higgs bosons which largely simplifies the analysis since only the pseudoscalar Higgs boson contributes to the decay amplitudes of the processes under consideration. It is worth noticing that a restricted set of these $\tau$ decays were considered in Ref. 10] in the 2HDM-III but without assuming CP conservation.

This paper is organized as follows. In section 2, we provide the Yukawa interactions for the neutral Higgs bosons. In section 3, we derive the LFV effective four-fermion hamiltonian and we provide the numerical values of couplings relevant for our calculations. The formulae needed to evaluate the two-body decay rates are given in section 4; from a comparison with the experimental upper limits on branching fractions we derive the bounds on flavor violating couplings. Finally, in section 5, we present our conclusions.

\section{FLAVOR VIOLATION IN THE 2HDM-III}

All lepton flavor violating processes in the SM have vanishing decay probabilities at the tree level and even at 1-loop level they are completely negligible [2] and beyond the reach of present and planned experiments. Therefore, flavor violation at a level accessible to experiments can be expected only in the framework of New Physics scenarios. In this section we discuss the Yukawa 
couplings of fermions in the 2HDM-III which can induce flavor-changing neutral current processes both in the leptonic and quark sectors. We also provide numerical values of couplings that enter the hadronic matrix elements.

In the next subsections we shall derive the Yukawa interactions of the scalar $\left(h^{0}, H^{0}\right)$ and pseudoscalar $\left(A^{0}\right)$ neutral Higgs bosons with the charged lepton and quark sectors of the 2HDMIII by assuming four-texture mass matrices [11, 12, 13].

\section{A. The charged lepton sector}

The Yukawa lagrangian of the 2HDM-III for the charged lepton sector is given by:

$$
\mathcal{L}_{Y}^{l}=Y_{1 i j}^{l} \bar{L}_{i} \Phi_{1} l_{R j}+Y_{2 i j}^{l} \bar{L}_{i} \Phi_{2} l_{R j}+\text { h.c. }, .
$$

where $\Phi_{1,2}=\left(\phi_{1,2}^{+}, \phi_{1,2}^{0}\right)^{T}$ denote the Higgs doublets and $L_{i}$ denote the doublet of left-handed leptons. The specific choices for the Yukawa matrices $Y_{1,2}^{l}$ define the versions of the 2HDM known as type I, II or III.

After spontaneous symmetry breaking the charged lepton mass matrix is given by,

$$
M_{l}=\frac{1}{\sqrt{2}}\left(v_{1} Y_{1}^{l}+v_{2} Y_{2}^{l}\right)
$$

Now we assume that both Yukawa matrices $Y_{1}^{l}$ and $Y_{2}^{l}$ have the four-texture form and are Hermitian; following the conventions of [14], the lepton mass matrix is then written as:

$$
M_{l}=\left(\begin{array}{ccc}
0 & C_{l} & 0 \\
C_{l}^{*} & \tilde{B}_{l} & B_{l} \\
0 & B_{l}^{*} & A_{l}
\end{array}\right),
$$

such that when $\tilde{B}_{l} \rightarrow 0$ one recovers the six-texture form. We will also consider the following hierarchy, $\left|A_{l}\right| \gg\left|\tilde{B}_{l}\right|,\left|B_{l}\right|,\left|C_{l}\right|$, which is supported by the observed fermion masses in the SM.

Because of the Hermiticity condition, both $\tilde{B}_{l}$ and $A_{l}$ are real parameters, while the phases $\Phi_{B, C}$ of $C_{l}$ and $B_{l}$, can be removed from the mass matrix $M_{l}$ by defining: $M_{l}=P^{\dagger} \tilde{M}_{l} P$, where $P=\operatorname{diag}\left[1, e^{i \Phi_{C}}, e^{i\left(\Phi_{B}+\Phi_{C}\right)}\right]$, and the mass matrix $\tilde{M}_{l}$ includes only the real parts of $M_{l}$. The diagonalization of $\tilde{M}$ is then obtained by means of an orthogonal matrix $O$, such that the diagonal mass matrix is $\bar{M}_{l}=O^{T} \tilde{M}_{l} O$. 
The lagrangian (1) can be expanded in terms of the mass-eigenstates for the neutral $\left(h^{0}, H^{0}, A^{0}\right)$ and charged Higgs bosons $\left(H^{ \pm}\right)$. The interactions of the neutral Higgs bosons are then given by,

$$
\begin{aligned}
\mathcal{L}_{Y}^{l}=\frac{g}{2}\left(\frac{m_{i}}{m_{W}}\right) & \bar{l}_{i}\left\{\left[\frac{\cos \alpha}{\cos \beta} \delta_{i j}+\frac{\sqrt{2} \sin (\alpha-\beta)}{g \cos \beta}\left(\frac{m_{W}}{m_{i}}\right) \tilde{Y}_{2 i j}^{l}\right] H^{0}\right. \\
+ & {\left[-\frac{\sin \alpha}{\cos \beta} \delta_{i j}+\frac{\sqrt{2} \cos (\alpha-\beta)}{g \cos \beta}\left(\frac{m_{W}}{m_{i}}\right) \tilde{Y}_{2 i j}^{l}\right] h^{0} } \\
+ & \left.i\left[-\tan \beta \delta_{i j}+\frac{\sqrt{2}}{g \cos \beta}\left(\frac{m_{W}}{m_{i}}\right) \tilde{Y}_{2 i j}^{l}\right] \gamma^{5} A^{0}\right\} l_{j}+\text { h.c. }
\end{aligned}
$$

where $\alpha$ denotes the mixing angle which is used to define the physical mass eigenstates $h^{0}$ and $H^{0}$ in the CP-even Higgs sector,and $\tan \beta$ is the ratio of vacuum expectation values of the two Higgs doublets [15]. In the above expression, the terms proportional to $\delta i j$ corresponds to the modification of the $2 \mathrm{HDM}-\mathrm{II}$ over the SM result, while the terms proportional to $\tilde{Y}_{2}^{l}$ denotes the new contribution from the 2HDM-III. Thus, the physical fermion-Higgs couplings respect CPinvariance, despite the fact that the Yukawa matrices include complex phases; this follows because of the Hermiticity conditions imposed on both $Y_{1}^{l}$ and $Y_{2}^{l}$ matrices.

The correction terms to the lepton flavor conserving (LFC) and violating (LFV) couplings, depend on the rotated matrix $\tilde{Y}_{2}^{l}=O^{T} P Y_{2}^{l} P^{\dagger} O$. We can derive $\tilde{Y}_{2}^{l}$, by assuming that $Y_{2}^{l}$ has a four-texture form, namely:

$$
Y_{2}^{l}=\left(\begin{array}{ccc}
0 & C_{2} & 0 \\
C_{2}^{*} & \tilde{B}_{2} & B_{2} \\
0 & B_{2}^{*} & A_{2}
\end{array}\right), \quad\left|A_{2}\right| \gg\left|\tilde{B}_{2}\right|,\left|B_{2}\right|,\left|C_{2}\right|
$$

Since the orthogonal matrix that diagonalizes the real matrix $\tilde{M}_{l}$ with the four-texture form, has the form:

$$
O=\left(\begin{array}{ccc}
\sqrt{\frac{\lambda_{2} \lambda_{3}\left(A-\lambda_{1}\right)}{A\left(\lambda_{2}-\lambda_{1}\right)\left(\lambda_{3}-\lambda_{1}\right)}} & \eta \sqrt{\frac{\lambda_{1} \lambda_{3}\left(\lambda_{2}-A\right)}{A\left(\lambda_{2}-\lambda_{1}\right)\left(\lambda_{3}-\lambda_{2}\right)}} & \sqrt{\frac{\lambda_{1} \lambda_{2}\left(A-\lambda_{3}\right)}{A\left(\lambda_{3}-\lambda_{1}\right)\left(\lambda_{3}-\lambda_{2}\right)}} \\
-\eta \sqrt{\frac{\lambda_{1}\left(\lambda_{1}-A\right)}{\left(\lambda_{2}-\lambda_{1}\right)\left(\lambda_{3}-\lambda_{1}\right)}} & \sqrt{\frac{\lambda_{2}\left(A-\lambda_{2}\right)}{\left(\lambda_{2}-\lambda_{1}\right)\left(\lambda_{3}-\lambda_{2}\right)}} & \sqrt{\frac{\lambda_{3}\left(\lambda_{3}-A\right)}{\left(\lambda_{3}-\lambda_{1}\right)\left(\lambda_{3}-\lambda_{2}\right)}} \\
\eta \sqrt{\frac{\lambda_{1}\left(A-\lambda_{2}\right)\left(A-\lambda_{3}\right)}{A\left(\lambda_{2}-\lambda_{1}\right)\left(\lambda_{3}-\lambda_{1}\right)}} & -\sqrt{\frac{\lambda_{2}\left(A-\lambda_{1}\right)\left(\lambda_{3}-A\right)}{A\left(\lambda_{2}-\lambda_{1}\right)\left(\lambda_{3}-\lambda_{2}\right)}} & \sqrt{\frac{\lambda_{3}\left(A-\lambda_{1}\right)\left(A-\lambda_{2}\right)}{A\left(\lambda_{3}-\lambda_{1}\right)\left(\lambda_{3}-\lambda_{2}\right)}}
\end{array}\right),
$$

where $m_{e}=m_{1}=\left|\lambda_{1}\right|, m_{\mu}=m_{2}=\left|\lambda_{2}\right|, m_{\tau}=m_{3}=\left|\lambda_{3}\right|$ and $\eta=\lambda_{2} / m_{2}$, then the rotated form of $\tilde{Y}_{2}^{l}$ acquires the general form,

$$
\begin{aligned}
\tilde{Y}_{2}^{l}= & O^{T} P Y_{2}^{l} P^{\dagger} O \\
& =\left(\begin{array}{ccc}
\left(\tilde{Y}_{2}^{l}\right)_{11} & \left(\tilde{Y}_{2}^{l}\right)_{12} & \left(\tilde{Y}_{2}^{l}\right)_{13} \\
\left(\tilde{Y}_{2}^{l}\right)_{21} & \left(\tilde{Y}_{2}^{l}\right)_{22} & \left(\tilde{Y}_{2}^{l}\right)_{23} \\
\left(\tilde{Y}_{2}^{l}\right)_{31} & \left(\tilde{Y}_{2}^{l}\right)_{32} & \left(\tilde{Y}_{2}^{l}\right)_{33}
\end{array}\right)
\end{aligned}
$$


The full expressions for the resulting entries of this matrix have a complicated form. To derive a convenient approximation, we will consider that the elements of the Yukawa matrix $Y_{2}^{l}$ exhibit the same hierarchy as the full mass matrix, namely:

$$
\begin{aligned}
& C_{2}=c_{2} \sqrt{\frac{m_{1} m_{2} m_{3}}{A}}, \\
& B_{2}=b_{2} \sqrt{\left(A-\lambda_{2}\right)\left(m_{3}-A\right)}, \\
& \tilde{B}_{2}=\tilde{b}_{2}\left(m_{3}-A+\lambda_{2}\right) \\
& A_{2}=a_{2} A .
\end{aligned}
$$

In order to keep the same hierarchy for the elements of the mass matrix, it is found that $A$ must satisfy the condition $\left(m_{3}-m_{2}\right) \leq A \leq m_{3}$. Thus, we propose the following relation for $A$ :

$$
A=m_{3}(1-\beta z)
$$

where $z=m_{2} / m_{3} \ll 1$ and $0 \leq \beta \leq 1$.

If we now introduce the dimensionless matrix $\tilde{\chi}$, we get:

$$
\begin{aligned}
\left(\tilde{Y}_{2}^{l}\right)_{i j} & =\frac{\sqrt{m_{i} m_{j}}}{v} \tilde{\chi}_{i j} \\
& =\frac{\sqrt{m_{i} m_{j}}}{v} \chi_{i j} e^{\vartheta_{i j}} .
\end{aligned}
$$

This expression differs from the usual Cheng-Sher Ansatz [16] not only because of the appearance of the complex phases, but also in the form of the real parts $\chi_{i j}=\left|\tilde{\chi}_{i j}\right|$.

If we expand in powers of $z$, the entries of the matrix $\tilde{\chi}$ become:

$$
\begin{aligned}
& \tilde{\chi}_{11}=\left[\tilde{b}_{2}-\left(c_{2}^{*} e^{i \Phi_{C}}+c_{2} e^{-i \Phi_{C}}\right)\right] \eta+\left[a_{2}+\tilde{b}_{2}-\left(b_{2}^{*} e^{i \Phi_{B}}+b_{2} e^{-i \Phi_{B}}\right)\right] \beta \\
& \left.\tilde{\chi}_{12}=\left(c_{2} e^{-i \Phi_{C}}-\tilde{b}_{2}\right)-\eta\left[a_{2}+\tilde{b}_{2}-\left(b_{2}^{*} e^{i \Phi_{B}}+b_{2} e^{-i \Phi_{B}}\right)\right] \beta\right] \\
& \tilde{\chi}_{13}=\left(a_{2}-b_{2} e^{-i \Phi_{B}}\right) \eta \sqrt{\beta} \\
& \tilde{\chi}_{22}=\tilde{b}_{2} \eta+\left[a_{2}+\tilde{b}_{2}-\left(b_{2}^{*} e^{i \Phi_{B}}+b_{2} e^{-i \Phi_{B}}\right)\right] \beta \\
& \tilde{\chi}_{23}=\left(b_{2} e^{-i \Phi_{B}}-a_{2}\right) \sqrt{\beta} \\
& \tilde{\chi}_{33}=a_{2}
\end{aligned}
$$

As it can be easily checked, the diagonal elements $\tilde{\chi}_{i i}$ are real and the phases appear in the off-diagonal elements; we note that these elements are not constrained by current low-energy phenomena. Furthermore the LFV couplings satisfy some relations, such as: $\left|\tilde{\chi}_{23}\right|=\left|\tilde{\chi}_{13}\right|$, which simplifies somehow the number of free parameters. 
Finally, in order to perform phenomenological studies it is found convenient to rewrite the lagrangian given in Eq. (44) in terms of the $\tilde{\chi}_{i j}$ 's as follows:

$$
\begin{aligned}
\mathcal{L}_{Y}^{l}= & \frac{g}{2} \bar{l}_{i}\left[\left(\frac{m_{i}}{m_{W}}\right) \frac{\cos \alpha}{\cos \beta} \delta_{i j}+\frac{\sin (\alpha-\beta)}{\sqrt{2} \cos \beta}\left(\frac{\sqrt{m_{i} m_{j}}}{m_{W}}\right) \tilde{\chi}_{i j}\right] l_{j} H^{0} \\
& +\frac{g}{2} \bar{l}_{i}\left[-\left(\frac{m_{i}}{m_{W}}\right) \frac{\sin \alpha}{\cos \beta} \delta_{i j}+\frac{\cos (\alpha-\beta)}{\sqrt{2} \cos \beta}\left(\frac{\sqrt{m_{i} m_{j}}}{m_{W}}\right) \tilde{\chi}_{i j}\right] l_{j} h^{0} \\
& +\frac{i g}{2} \bar{l}_{i}\left[-\left(\frac{m_{i}}{m_{W}}\right) \tan \beta \delta_{i j}+\frac{1}{\sqrt{2} \cos \beta}\left(\frac{\sqrt{m_{i} m_{j}}}{m_{W}}\right) \tilde{\chi}_{i j}\right] \gamma^{5} l_{j} A^{0}+\text { h.c. },
\end{aligned}
$$

where, unlike the Cheng-Sher Ansatz, $\tilde{\chi}_{i j}(i \neq j)$ are now complex parameters.

\section{B. The quark sector}

The Yukawa lagrangian for the quark fields in the 2HDM-III model is written as:

$$
\mathcal{L}_{Y}^{q}=Y_{1}^{u} \bar{Q}_{L} \Phi_{1} u_{R}+Y_{2}^{u} \bar{Q}_{L} \Phi_{2} u_{R}+Y_{1}^{d} \bar{Q}_{L} \Phi_{1} d_{R}+Y_{2}^{d} \bar{Q}_{L} \Phi_{2} d_{R}+h . c .
$$

where $Q_{L}$ denote the doublet of left-handed quarks. Again, the specific choices for the Yukawa matrices $Y_{1,2}^{q}(q=u, d)$ define the versions of the 2HDM known as type I, II or III.

Following a similar procedure as in the case of the charged leptons we can derive the Yukawa couplings for the physical neutral Higgs bosons $\left(h^{0}, H^{0}, A^{0}\right)$. If we introduce the dimensionless matrix $\tilde{\chi}_{q q^{\prime}}$ :

$$
\begin{aligned}
\left(\tilde{Y}_{2}^{q}\right)_{q q^{\prime}} & =\frac{\sqrt{m_{q} m_{q^{\prime}}}}{v} \tilde{\chi}_{q q^{\prime}} \\
& =\frac{\sqrt{m_{q} m_{q^{\prime}}}}{v} \chi_{q q^{\prime}} e^{i \vartheta_{q q^{\prime}}}
\end{aligned}
$$

to replace the Yukawa matrices in the rotated basis, we get the explicit form of the Yukawa couplings for the neutral Higgs bosons:

$$
\begin{aligned}
\mathcal{L}_{Y}^{q}= & \frac{g}{2} \bar{d}\left[\left(\frac{m_{d}}{m_{W}}\right) \frac{\cos \alpha}{\cos \beta} \delta_{d d^{\prime}}+\frac{\sin (\alpha-\beta)}{\sqrt{2} \cos \beta}\left(\frac{\sqrt{m_{d} m_{d^{\prime}}}}{m_{W}}\right) \tilde{\chi}_{d d^{\prime}}\right] d^{\prime} H^{0} \\
& +\frac{g}{2} \bar{d}\left[-\left(\frac{m_{d}}{m_{W}}\right) \frac{\sin \alpha}{\cos \beta} \delta_{d d^{\prime}}+\frac{\cos (\alpha-\beta)}{\sqrt{2} \cos \beta}\left(\frac{\sqrt{m_{d} m_{d^{\prime}}}}{m_{W}}\right) \tilde{\chi}_{d d^{\prime}}\right] d^{\prime} h^{0} \\
& +\frac{i g}{2} \bar{d}\left[-\left(\frac{m_{d}}{m_{W}}\right) \tan \beta \delta_{d d^{\prime}}+\frac{1}{\sqrt{2} \cos \beta}\left(\frac{\sqrt{m_{d} m_{d^{\prime}}}}{m_{W}}\right) \tilde{\chi}_{d d^{\prime}}\right] \gamma^{5} d^{\prime} A^{0} \\
& +\frac{g}{2} \bar{u}\left[\left(\frac{m_{u}}{m_{W}}\right) \frac{\sin \alpha}{\sin \beta} \delta_{u u^{\prime}}-\frac{\sin (\alpha-\beta)}{\sqrt{2} \sin \beta}\left(\frac{\sqrt{m_{u} m_{u^{\prime}}}}{m_{W}}\right) \tilde{\chi}_{u u^{\prime}}\right] u^{\prime} H^{0} \\
& +\frac{g}{2} \bar{u}\left[\left(\frac{m_{u}}{m_{W}}\right) \frac{\cos \alpha}{\sin \beta} \delta_{u u^{\prime}}-\frac{\cos (\alpha-\beta)}{\sqrt{2} \sin \beta}\left(\frac{\sqrt{m_{u} m_{u^{\prime}}}}{m_{W}}\right) \tilde{\chi}_{u u^{\prime}}\right] u^{\prime} h^{0} \\
& +\frac{i g}{2} \bar{u}\left[-\left(\frac{m_{u}}{m_{W}}\right) \cot \beta \delta_{u u^{\prime}}+\frac{1}{\sqrt{2} \sin \beta}\left(\frac{\sqrt{m_{u} m_{u^{\prime}}}}{m_{W}}\right) \tilde{\chi}_{u u^{\prime}}\right] \gamma^{5} u^{\prime} A^{0}+h . c .,
\end{aligned}
$$


where $d$ (respectively, $u$ ) stands for down-type (up-type) quarks.

In our discussion above, the flavor changing couplings are contained in the entries $\tilde{\chi}_{f f^{\prime}}$; these dimensionless parameters are expected to be of order one or below and we will consider that the bounds obtained from data are significant if this turns out to be the case. In our calculations below, we will neglect the contributions of $\tilde{\chi}_{f f}$ in the flavor conserving vertices. Note that processes with flavor violation at both vertices, like $\tau \rightarrow \mu K_{S}$ or $B^{0} \rightarrow e \mu$, are allowed to occur in our model. However, we will not consider this type of decays because data allows to bound the product of flavor violating couplings and not these couplings themselves.

It is interesting to note that in the above version of the 2HDM-III, the assumption that the Yukawa matrices are Hermitean leads to CP conservation, and this implies that the scalar (pseudoscalar) Higgs bosons couple only to scalar (pseudoscalar) fermionic currents. This will greatly simplify the analysis of this paper since only the pseudoscalar Higgs boson will contribute to the decays of our interest.

\section{EFFECTIVE HAMILTONIAN AND COUPLING CONSTANTS}

The Feynman graphs corresponding to the two-body decays of our interest are mediated by a pseudoscalar Higgs boson $A^{0}$ and are shown in Figure 1.

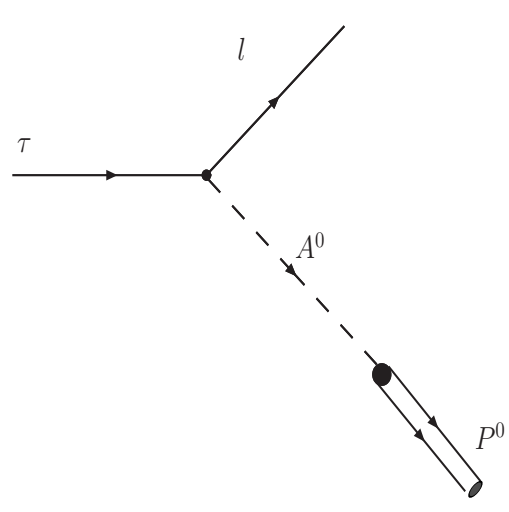

(a)

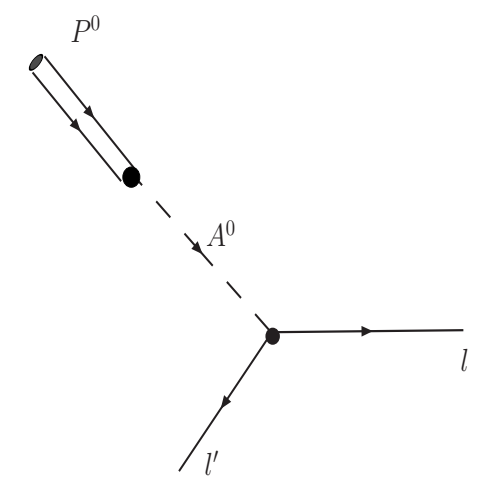

(b)

FIG. 1: Two-body decays mediated by the pseudoscalar Higgs boson $A^{0}$ : (a) tau lepton decays and, (b) Pseudoscalar meson decays.

In the local four-fermion approximation, which is valid for the energy scales involved in the 
decays under consideration, it is more convenient to work with the effective hamiltonian given by

$$
\mathcal{H}=\sqrt{2} G_{F}\left(\frac{m_{W}^{2}}{m_{A^{0}}^{2}}\right) g_{A l l^{\prime}} \bar{\psi}_{l^{\prime}} \gamma_{5} \psi_{l} \times\left[\sum_{q, q^{\prime}} g_{A q q^{\prime}} \bar{q}^{\prime} \gamma_{5} q\right]+\text { h.c. }
$$

where the coupling constants $g_{A f f^{\prime}}$ can be easily identified from the interaction lagrangians given in eqs. (1) and (2). The above interaction hamiltonian allows us to cover all the cases for flavor violation at the lepton and quark vertices as studied this paper.

The relevant hadronic matrix elements that will enter in our calculations are the following:

$$
\left(m_{q^{\prime}}+m_{q}\right)\left\langle 0\left|i \overline{q^{\prime}} \gamma_{5} q\right| P\right\rangle=h_{P}^{q}
$$

where $q, q^{\prime}$ denote the quarks $\left(q^{\prime} \neq q\right.$ only when flavor violation occurs at the meson vertex). Note that the $h_{P}^{q}$ constants can be directly related to the usual pseudoscalar meson decay constants $f_{P}$ (namely, $h_{P}=f_{P} m_{P}^{2}$ for all the cases except the $\pi^{0}, \eta, \eta^{\prime}$ mesons).

In this work we will use the isospin limit where $m_{u}=m_{d}=3.3 \mathrm{MeV}$. For the other quark masses we will use [17]: $m_{s}=0.104, m_{c}=1.27, m_{b}=4.2 \mathrm{GeV}$. In our numerical evaluations, we will use the following values of the decay constants of the unflavored mesons [18] (here $q=u, d$ ):

$$
\begin{aligned}
h_{\pi}^{q} & =2.38 \times 10^{-3} \mathrm{GeV}^{3}, \\
h_{\eta}^{q} & =2.0 \times 10^{-3} \mathrm{GeV}^{3}, \\
h_{\eta^{\prime}}^{q} & =1.6 \times 10^{-3} \mathrm{GeV}^{3}, \\
h_{\eta}^{s} & =-53 \times 10^{-3} \mathrm{GeV}^{3}, \\
h_{\eta^{\prime}}^{s} & =65 \times 10^{-3} \mathrm{GeV}^{3} .
\end{aligned}
$$

Equivalently, we can use information on the pseudoscalar decay constants from Refs. [17, 19, 20, 21]:

$$
\begin{aligned}
& f_{\pi^{0}}=(130 \pm 5) \mathrm{MeV} \\
& f_{K^{0}}=(155.5 \pm 0.9) \mathrm{MeV} \\
& f_{D^{0}}=(205.8 \pm 8.9) \mathrm{MeV} \\
& f_{B^{0}}=(216 \pm 22) \mathrm{MeV}, \\
& f_{B_{s}}=(260 \pm 29) \mathrm{MeV} .
\end{aligned}
$$

The quark content of unflavored mesons are the following:

$$
\begin{aligned}
\pi^{0} & =\frac{1}{\sqrt{2}}(\bar{u} u-\bar{d} d) \\
\eta & =\eta_{8} \cos \theta_{P}-\eta_{1} \sin \theta_{P} \\
\eta^{\prime} & =\eta_{8} \sin \theta_{P}+\eta_{1} \cos \theta_{P}
\end{aligned}
$$


where $\eta_{8,1}$ denote the octet and singlet isoscalar mesons and $\theta_{P} \approx-20^{\circ}$ is the mixing angle of pseudoscalar mesons.

\section{FLAVOR VIOLATION IN PRODUCTION/DECAY OF $P^{0}$ MESONS}

In this section we study the coupling of a pseudoscalar meson $P^{0}$ to a leptonic neutral current. We consider that the flavor change can occur at either the hadronic or leptonic vertex. When we compare our results with the current experimental bounds on branching fractions, we derive the constraints on the relevant flavor changing Yukawa couplings of the $A^{0}$ Higgs boson.

The decay widths in the case of $\tau$ lepton decays are given by:

$$
\begin{aligned}
\Gamma(\tau \rightarrow l P)= & \frac{G_{F}^{2}}{8 \pi}\left(\frac{m_{W}}{m_{A^{0}}}\right)^{4}\left[\left(m_{\tau}-m_{l}\right)^{2}-m_{P}^{2}\right] \frac{\lambda^{1 / 2}\left(m_{\tau}^{2}, m_{l}^{2}, m_{P}^{2}\right)}{m_{\tau^{3}}} \\
& \times g_{A \tau l}^{2}\left|\left\langle P\left|\sum_{q, q^{\prime}} g_{A q q^{\prime}} \bar{q}^{\prime} \gamma_{5} q\right| 0\right\rangle\right|^{2},
\end{aligned}
$$

while the corresponding widths for meson decays are:

$$
\begin{aligned}
\Gamma\left(P \rightarrow l l^{\prime}\right)= & \frac{G_{F}^{2}}{4 \pi}\left(\frac{m_{W}}{m_{A^{0}}}\right)^{4}\left[m_{P}^{2}-\left(m_{l}-m_{l^{\prime}}\right)^{2}\right] \cdot \frac{\lambda^{1 / 2}\left(m_{P}^{2}, m_{l}^{2}, m_{l^{\prime}}^{2}\right)}{m_{P}^{3}} \\
& \times g_{A l l^{\prime}}^{2}\left|\left\langle 0\left|\sum_{q, q^{\prime}} g_{A q q^{\prime}} \overline{q^{\prime}} \gamma_{5} q\right| P\right\rangle\right|^{2} .
\end{aligned}
$$

In the above expressions we have defined $\lambda(x, y, z)=x^{2}+y^{2}+z^{2}-2 x y-2 x z-2 y z$.

The hadronic matrix elements required for our numerical evaluations are the following:

$$
\begin{aligned}
\langle 0|O| \pi\rangle & =\frac{i}{\sqrt{2}} \frac{f_{\pi} m_{\pi}^{2}}{\left(m_{u}+m_{d}\right)}\left(g_{\text {Auu }}-g_{\text {Add }}\right) \\
\langle 0|O| \eta\rangle & =-\frac{i}{\sqrt{2}}\left[\left(g_{A u u}+g_{A d d}\right) \frac{h_{\eta}^{q}}{\left(m_{u}+m_{d}\right)}+\sqrt{2} g_{A s s} \frac{h_{\eta}^{s}}{2 m_{s}}\right] \\
\left\langle 0|O| \eta^{\prime}\right\rangle & =-\frac{i}{\sqrt{2}}\left[\left(g_{A u u}+g_{A d d}\right) \frac{h_{\eta^{\prime}}^{q}}{\left(m_{u}+m_{d}\right)}+\sqrt{2} g_{A s s} \frac{h_{\eta^{\prime}}^{s}}{2 m_{s}}\right] \\
\left\langle 0|O| D^{0}\right\rangle & =-i g_{A u c} \frac{f_{D} m_{D}^{2}}{m_{c}+m_{u}} \\
\left\langle 0|O| B^{0}\right\rangle & =-i g_{A d b} \frac{f_{B} m_{B}^{2}}{m_{b}+m_{d}} \\
\left\langle 0|O| B_{s}\right\rangle & =-i g_{A s b} \frac{f_{B_{s}} m_{B_{s}}^{2}}{m_{b}+m_{s}},
\end{aligned}
$$

where the operator $O$ is defined by $O \equiv \sum_{i} g_{A i j} \bar{q}_{j} \gamma_{5} q_{i}$ and the values of quark masses and decay constants were given in the previous section. 
$g_{A i j}$ is the coupling of the Higgs neutral boson to a fermion pair $i j$ and in our case are given as follows:

$$
\begin{gathered}
g_{A l l^{\prime}}=\frac{i g}{2}\left[-\left(\frac{m_{l}}{m_{W}}\right) \tan \beta \delta_{l l^{\prime}}+\frac{1}{\sqrt{2} \cos \beta}\left(\frac{\sqrt{m_{l} m_{l^{\prime}}}}{m_{W}}\right) \tilde{\chi}_{l l^{\prime}}\right], \\
g_{A u u^{\prime}}=\frac{i g}{2}\left[-\left(\frac{m_{u}}{m_{W}}\right) \cot \beta \delta_{u u^{\prime}}+\frac{1}{\sqrt{2} \sin \beta}\left(\frac{\sqrt{m_{u} m_{u^{\prime}}}}{m_{W}}\right) \tilde{\chi}_{u u^{\prime}}\right],
\end{gathered}
$$

and

$$
g_{A d d^{\prime}}=\frac{i g}{2}\left[-\left(\frac{m_{d}}{m_{W}}\right) \tan \beta \delta_{d d^{\prime}}+\frac{1}{\sqrt{2} \cos \beta}\left(\frac{\sqrt{m_{d} m_{d^{\prime}}}}{m_{W}}\right) \tilde{\chi}_{d d^{\prime}}\right]
$$

where $l, l^{\prime}=e, \mu, \tau ; u, u^{\prime}=u, c, t$; and $d, d^{\prime}=d, s, b$.

In order to derive the bounds on the New Physics couplings, we use the upper limits on the branching ratios reported by the PDG [17]. In Tables [, II and III, we show our results as upper bounds on the product of leptonic and quark couplings normalized to the square of the Higgs boson $A^{0}$ for $\tau$ lepton and pseudoscalar meson decays. These expressions look rather long, but they can be useful to easily implement future updates. Tables $\llbracket$ and $\Pi$ contain the upper bounds on LFV couplings, while Table III refers to the bounds on the flavor changing quark couplings. A comparison of results from Tables I and II shows that experimental data on $\tau$ lepton decays highly constrain the $\tau l$ LF couplings. Conversely we observe that decays of light mesons provide very poor constraints on $\mu$ LFV Yukawa couplings, despite the tightly upper limits on their branching ratios .

Using the definitions of the effective couplings $g_{A f f^{\prime}}$ and the approximations noticed in section II we can translate the bounds shown in Tables I-III on the dimensionless couplings $\tilde{\chi}_{f f^{\prime}}$. As we mentioned before, we will say that a constraint is significant if these dimensionless couplings turn out to be smaller that unity. In Figures 2, 3, 4 we show the bounds on the Yukawa couplings $\chi_{e \tau}=\left|\tilde{\chi}_{e \tau}\right|, \chi_{\mu \tau}=\left|\tilde{\chi}_{\mu \tau}\right|, \chi_{e \mu}=\left|\tilde{\chi}_{e \mu}\right|$, as a function of $\tan \beta$ by taking $m_{A^{0}}=300 \mathrm{GeV}$, respectively. As we have mentioned in section II, our imposition of the Hermiticity conditions on the fermion mass matrices which implies CP-conservation in the Yukawa sector allows us to derive bounds directly on the couplings of the pseudoscalar Higgs boson. As we observe in Figs. 24, these bounds become more restrictive when $\tan \beta$ increases. Conversely, these bounds are relaxed for 


\begin{tabular}{|c|cc|c|}
\hline Process & BR (PDG 2008)[17] & Upper limit \\
\hline$\tau^{-} \rightarrow \pi^{0} e^{-}$ & $<8.0 \times 10^{-8}$ & $\left|\frac{g_{A \tau e}}{m_{A^{0}}^{2}} \frac{1}{\sqrt{2}} \frac{f_{\pi} m_{\pi}^{2}}{\left(m_{u}+m_{d}\right)}\left(g_{A u u}-g_{A d d}\right)\right|<2.14 \times 10^{-8}$ \\
$\tau^{-} \rightarrow \pi^{0} \mu^{-}$ & $<1.1 \times 10^{-7}$ & $\left|\frac{g_{A \tau \mu}}{m_{A^{0}}^{2}} \frac{1}{\sqrt{2}} \frac{f_{\pi} m_{\pi}^{2}}{\left(m_{u}+m_{d}\right)}\left(g_{A u u}-g_{A d d}\right)\right|<2.67 \times 10^{-8}$ \\
$\tau^{-} \rightarrow \eta e^{-}$ & $<9.2 \times 10^{-8}$ & $\left|\frac{g_{A \tau e}}{m_{A^{0}}^{2}} \frac{1}{\sqrt{2}}\left[\left(g_{A u u}+g_{A d d}\right) \frac{h_{\eta}^{q}}{\left(m_{u}+m_{d}\right)}+\sqrt{2} g_{A s s} \frac{h_{\eta}^{s}}{2 m_{s}}\right]\right|<4.25 \times 10^{-8}$ \\
$\tau^{-} \rightarrow \eta \mu^{-}$ & $<6.5 \times 10^{-8}$ & $\frac{g_{A \tau \mu}}{m_{A^{0}}^{2}} \frac{1}{\sqrt{2}}\left[\left(g_{A u u}+g_{A d d}\right) \frac{h_{\eta}^{q}}{\left(m_{u}+m_{d}\right)}+\sqrt{2} g_{A s s} \frac{h_{\eta}^{s}}{2 m_{s}}\right] \mid<2.27 \times 10^{-8}$ \\
$\tau^{-} \rightarrow \eta^{\prime} e^{-}$ & $<1.6 \times 10^{-7}$ & $\frac{g_{A \tau e}}{m_{A^{0}}} \frac{1}{\sqrt{2}}\left[\left(g_{A u u}+g_{A d d}\right) \frac{h_{\eta^{\prime}}^{q}}{\left(m_{u}+m_{d}\right)}+\sqrt{2} g_{A s s} \frac{h_{\eta^{\prime}}^{s}}{2 m_{s}}\right] \mid<4.24 \times 10^{-8}$ \\
$\tau^{-} \rightarrow \eta^{\prime} \mu^{-}$ & $<1.3 \times 10^{-7}$ & $\frac{g_{A \tau \mu}}{m_{A^{0}}^{2}} \frac{1}{\sqrt{2}}\left[\left(g_{A u u}+g_{A d d}\right) \frac{h_{\eta^{\prime}}^{q}}{\left(m_{u}+m_{d}\right)}+\sqrt{2} g_{A s s} \frac{h_{\eta^{\prime}}^{s}}{2 m_{s}}\right]$ & $<4.19 \times 10^{-8}$ \\
\hline
\end{tabular}

TABLE I: LFV tau decays to a light pseudoscalar mesons.

\begin{tabular}{|c|cc|}
\hline Process & BR (PDG 2008) [17] & Upper limit \\
\hline$\pi^{0} \rightarrow \mu^{+} e^{-}$ & $<3.8 \times 10^{-10}$ & $\left|\frac{g_{A e \mu}}{m_{A^{0}}^{2}} \frac{1}{\sqrt{2}} \frac{f_{\pi} m_{\pi}^{2}}{\left(m_{u}+m_{d}\right)}\left(g_{A u u}-g_{A d d}\right)\right|<4.55 \times 10^{-2}$ \\
$\pi^{0} \rightarrow \mu^{-} e^{+}$ & $<3.4 \times 10^{-9}$ & $\left|\frac{g_{A e \mu}}{m_{A^{0}}^{2}} \frac{1}{\sqrt{2}} \frac{f_{\pi} m_{\pi}^{2}}{\left(m_{u}+m_{d}\right)}\left(g_{A u u}-g_{A d d}\right)\right|<0.14$ \\
$\eta \rightarrow \mu^{ \pm} e^{\mp}$ & $<6.0 \times 10^{-6}$ & $\left|\frac{g_{A e \mu}}{m_{A^{0}}^{2}} \frac{1}{\sqrt{2}}\left[\left(g_{A u u}+g_{A d d}\right) \frac{h_{\eta}^{q}}{\left(m_{u}+m_{d}\right)}+\sqrt{2} g_{A s s} \frac{h_{\eta}^{s}}{2 m_{s}}\right]\right|<14.81$ \\
$\eta^{\prime} \rightarrow \mu^{ \pm} e^{\mp}$ & $<4.7 \times 10^{-4}$ & $\frac{g_{A e \mu}}{m_{A^{0}}^{2}} \frac{1}{\sqrt{2}}\left[\left(g_{A u u}+g_{A d d}\right) \frac{h_{\eta^{\prime}}^{q}}{\left(m_{u}+m_{d}\right)}+\sqrt{2} g_{A s s} \frac{h_{\eta^{\prime}}^{s}}{2 m_{s}}\right] \mid<1.21 \times 10^{3}$ \\
\hline
\end{tabular}

TABLE II: LFV decays of light pseudoscalar mesons.

increasing values of the $A^{0}$ Higgs boson mass because these bounds are proportional to $m_{A^{0}}^{2}$. It is shown in Fig. 2 that the most restrictive bound on $\chi_{e \tau}$ is obtained from $\tau^{-} \rightarrow \eta^{\prime} e^{-}$decay, where we obtain $\chi_{e \tau}<134$ for $m_{A^{0}}=300 \mathrm{GeV}$ and $\tan \beta=50$. From Fig. 3 we observe that the most restrictive bound on $\chi_{\mu \tau}$ is obtained from $\tau^{-} \rightarrow \eta \mu^{-}$decay, with $\chi_{\mu \tau}<6.68$ for $m_{A^{0}}=300 \mathrm{GeV}$ and $\tan \beta=50$. We recall here that $\chi_{e \tau}=\chi_{\mu \tau}$ in the model under consideration [11, 12].

\begin{tabular}{|c|cc|}
\hline Process & BR(PDG 2008)[17] & Upper limit \\
\hline$B^{0} \rightarrow e^{+} e^{-}$ & $<1.3 \times 10^{-7}$ & $\left|\frac{g_{A e e}}{m_{A^{0}}^{2}} g_{A d b} \frac{f_{B} m_{B}^{2}}{m_{b}+m_{d}}\right|<9.17 \times 10^{-10}$ \\
$B^{0} \rightarrow \mu^{+} \mu^{-}$ & $<1.5 \times 10^{-8}$ & $\left|\frac{g_{A \mu \mu}}{m_{A^{0}}^{2}} g_{A d b} \frac{f_{B} m_{B}^{2}}{m_{b}+m_{d}}\right|<3.12 \times 10^{-10}$ \\
$B^{0} \rightarrow \tau^{+} \tau^{-}$ & $<4.1 \times 10^{-3}$ & $\left|\frac{g_{A \tau \tau}}{m_{A^{0}}^{2}} g_{A d b} \frac{f_{B} m_{B}^{2}}{m_{b}+m_{d}}\right|<2.20 \times 10^{-7}$ \\
$B_{s}^{0} \rightarrow e^{+} e^{-}$ & $<5,4 \times 10^{-5}$ & $\left|\frac{g_{A e e}}{m_{A 0}^{2}} g_{A s b} \frac{f_{B_{s}} m_{B_{s}}^{2}}{m_{b}+m_{s}}\right|<1.86 \times 10^{-8}$ \\
$B_{s}^{0} \rightarrow \mu^{+} \mu^{-}$ & $<4.7 \times 10^{-8}$ & $\left|\frac{g_{A \mu \mu}}{m_{A^{0}}^{2}} g_{A s b} \frac{f_{B_{s}} m_{B_{s}}}{m_{b}+m_{s}}\right|<5.49 \times 10^{-10}$ \\
$D^{0} \rightarrow e^{+} e^{-}$ & $<1.2 \times 10^{-6}$ & $\left|\frac{g_{A e e}}{m_{A^{0}}^{2}} g_{A u c} \frac{f_{D} m_{D}^{2}}{m_{c}+m_{u}}\right|<2.56 \times 10^{-8}$ \\
$D^{0} \rightarrow \mu^{+} \mu^{-}$ & $<1.3 \times 10^{-6}$ & $\left|\frac{g_{A \mu \mu}}{m_{A^{0}}^{2}} g_{A u c} \frac{f_{D} m_{D}^{2}}{m_{c}+m_{u}}\right|<2.68 \times 10^{-8}$ \\
\hline
\end{tabular}

TABLE III: Flavor violation in decays of pseudoscalar mesons. 


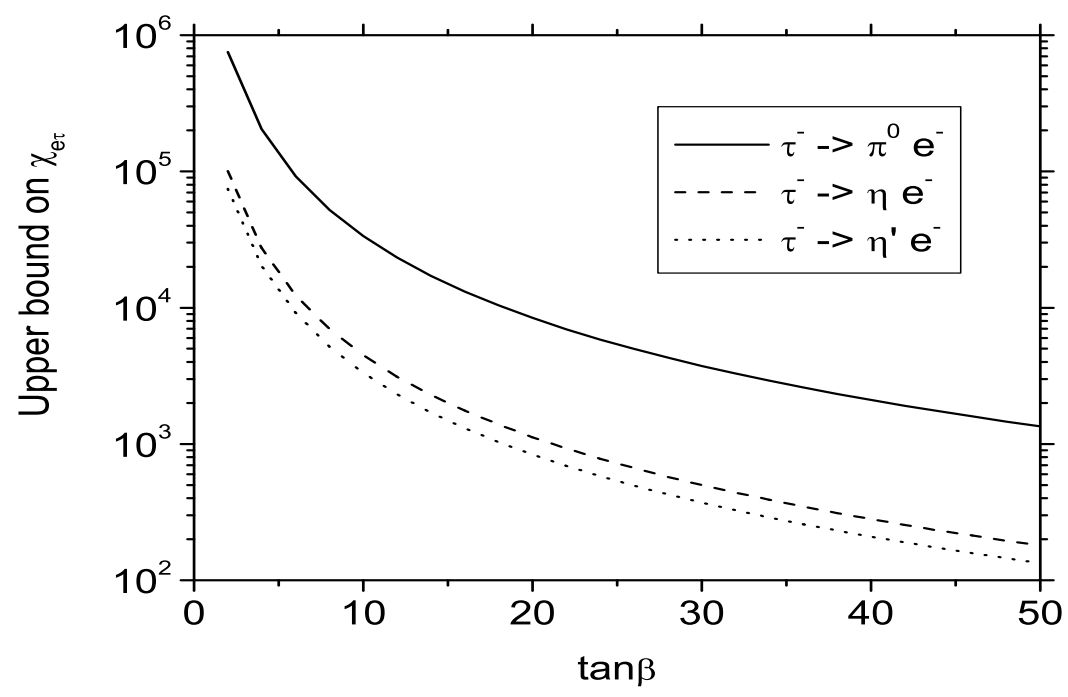

FIG. 2: Upper bound on $\chi_{e \tau}$ as a function of $\tan \beta$ for $m_{A^{0}}=300 \mathrm{GeV}$ (See Table \).

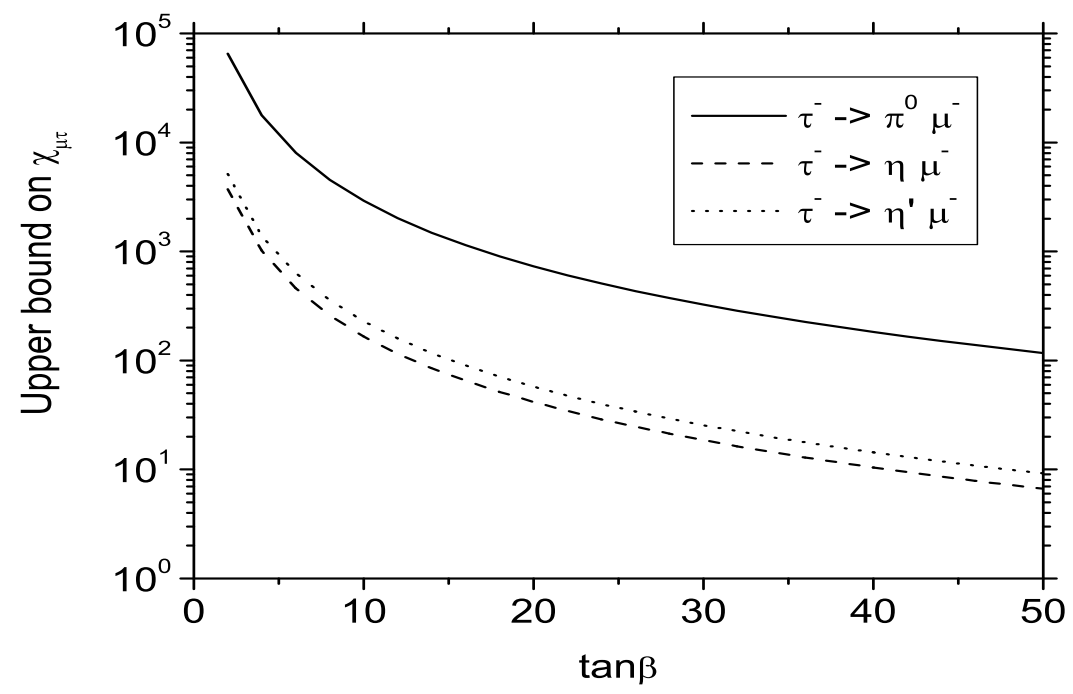

FIG. 3: Upper bound on $\chi_{\mu \tau}$ as a function of $\tan \beta$ for $m_{A^{0}}=300 \mathrm{GeV}$ (See Table 【).

In Figures 50 we show the bounds on the Yukawa couplings $\chi_{s b}=\left|\tilde{\chi}_{s b}\right|, \chi_{d b}=\left|\tilde{\chi}_{d b}\right|$, $\chi_{u c}=\left|\tilde{\chi}_{u c}\right|$ as a function of $\tan \beta$ by taking $m_{A^{0}}=300 \mathrm{GeV}$, respectively. As it can be seen in Fig. 5, the most restrictive bound on $\chi_{s b}$ is obtained from $B_{s}^{0} \rightarrow \mu^{-} \mu^{+}$decay, where we obtain $\chi_{s b}<1.40 \times 10^{-2}$ for $m_{A^{0}}=300 \mathrm{GeV}$ and $\tan \beta=50$. We observe in Fig. 6 that the most 


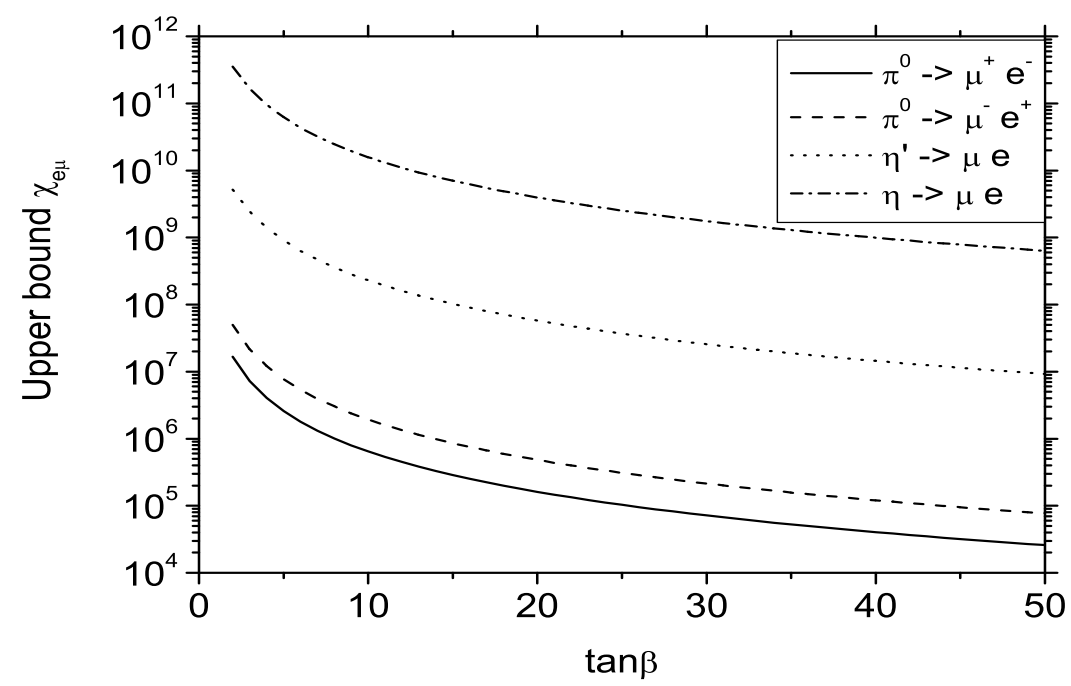

FIG. 4: Upper bound on $\chi_{e \mu}$ as a function of $\tan \beta$ for $m_{A^{0}}=300 \mathrm{GeV}$ (See Table II).

restrictive bound on $\chi_{d b}$ is obtained from $B^{0} \rightarrow \mu^{-} \mu^{+}$decay, we obtain $\chi_{d b}<4.01 \times 10^{-2}$ for $m_{A^{0}}=300 \mathrm{GeV}$ and $\tan \beta=50$. We recall here that $\chi_{d b}=\chi_{s b}$ in the model under consideration. Using the same input parameters for $\tan \beta$ and $m_{A^{0}}$, we observe in Fig. 7 that the most restrictive bound on $\chi_{u c}$ is obtained from $D^{0} \rightarrow \mu^{-} \mu^{+}$decay, namely $\chi_{u c}<O\left(10^{3}\right)$ which is indeed very poor. We should note that the upper bounds on the $\chi_{f f^{\prime}}$ quark couplings are rather conservative as long as the SM will also give a contribution via the usual quark mixing mechanism.

In the context of the same model used in this article, in Ref. [12] we have reported bounds on the $\chi_{s b}$ coupling, which are of the same order of magnitude than those presented here. However, we want to point out that the upper bounds reported in the present work have been obtained by using processes which involve only $A^{0}$-exchange contributions, while those reported in Ref. [12] have been gotten by using processes which involve $h^{0}$-exchange contributions. Hence, our bounds depend only on $\tan \beta$, while the bounds reported in Ref. [12] depend on both parameters, $\tan \beta$ and $\alpha$.

Finally, we can compare processes involving flavor violation at one and the two vertices by taking the ratio of the $R_{l l^{\prime}} \equiv \Gamma\left(P^{0} \rightarrow l^{+} l^{\prime-}\right) / \Gamma\left(P^{0} \rightarrow l^{+} l^{-}\right)$decay rates (here $\left.l \neq l^{\prime}\right)$. This ratio is independent of the hadronic parameters and of the $A^{0}$ boson mass, actually $R_{l l^{\prime}} \approx\left(m_{l^{\prime}} / 2 m_{l}\right)$. $\left(\chi_{l l^{\prime}} / \sin \beta\right)^{2}$ for heavy meson decays into light leptons. Thus, for large enough values of $\tan \beta$ 
(typically $\tan \beta \geq 5$ ) and under the assumption that $\chi_{l l^{\prime}} \leq 1, R_{\mu e}$ gets suppressed by a least a factor of the $e / \mu$ mass ratio.

\section{CONCLUSIONS}

In this paper we have studied the lepton flavor violation induced by the Yukawa couplings of neutral pseudoscalar Higgs boson $A^{0}$ of the 2HDM-III in the two-body decays of $\tau$ leptons and pseudoscalar mesons. Under the assumption that the Yukawa matrices are Hermitean which implies that $\mathrm{CP}$ is conserved by these interactions, we are able to get bounds on the flavor-violating Yukawa couplings of the pseudoscalar Higgs boson. Using present data we have found the strongest bounds for the lepton-flavor violating couplings in the case of $\tau$ lepton decays involving $\left(\eta, \eta^{\prime}\right)$ mesons. Clearly, improved experimental upper limits on these decays by one or two orders of magnitude will produce significant bounds on the LF violating couplings. We have also considered LF conserving (but FCNC quark coupling) decays of neutral mesons. In this case we are able to find very significant constraints on the flavor-changing quark couplings under the conservative assumption that the SM contribution is negligible. In particular, we find $\chi_{s b}<1.40 \times 10^{-2}$ and $\chi_{d b}<4.01 \times 10^{-2}$ for the typical values $m_{A^{0}}=300 \mathrm{GeV}$ and $\tan \beta=50$. We thus conclude that neutral pseudoscalar mesons produced in $\tau$ lepton two-body decays and their decays to charged lepton pairs, provide information on the flavor-violating couplings that is complementary to the ones from the CP-even Higgs bosons.

\section{ACKNOWLEDGMENTS}

This work was supported in part by Consejo Nacional de Ciencia y Tecnología (México).

[1] N. Cabibbo, Phys. Rev. Lett. 10, 531 (1963); M. Kobayashi and T. Maskawa, Prog. Theor. Phys. 49, 652 (1973).

[2] S. Bergmann and Y. Grossman, Phys. Rev. D 59, 093005 (1999) arXiv:hep-ph/9809524; S. Bergmann, Y. Grossman and D. M. Pierce, Phys. Rev. D 61, 053005 (2000) arXiv:hep-ph/9909390; A. Masiero, S. K. Vempati and O. Vives, New J. Phys. 6, 202 (2004) arXiv:hep-ph/0407325; G. Altarelli, F. Feruglio and I. Masina, Phys. Lett. B 472, 382 (2000) arXiv:hep-ph/9907532.

[3] J. A. Casas and A. Ibarra, arXiv:hep-ph/0109161. W. Rodejohann, Phys. Rev. D 62 (2000) 013011 arXiv:hep-ph/0003149. V. D. Barger, arXiv:hep-ph/0102052. 


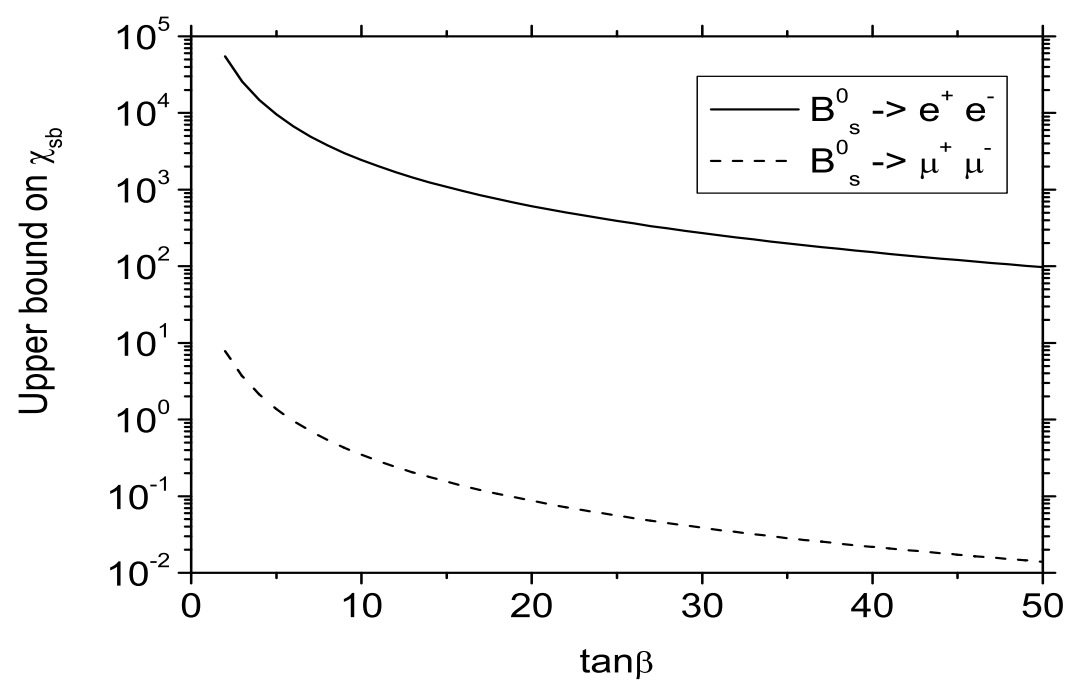

FIG. 5: Upper bound on $\chi_{s b}$ as a function of $\tan \beta$ for $m_{A^{0}}=300 \mathrm{GeV}$ (See Table 【II).

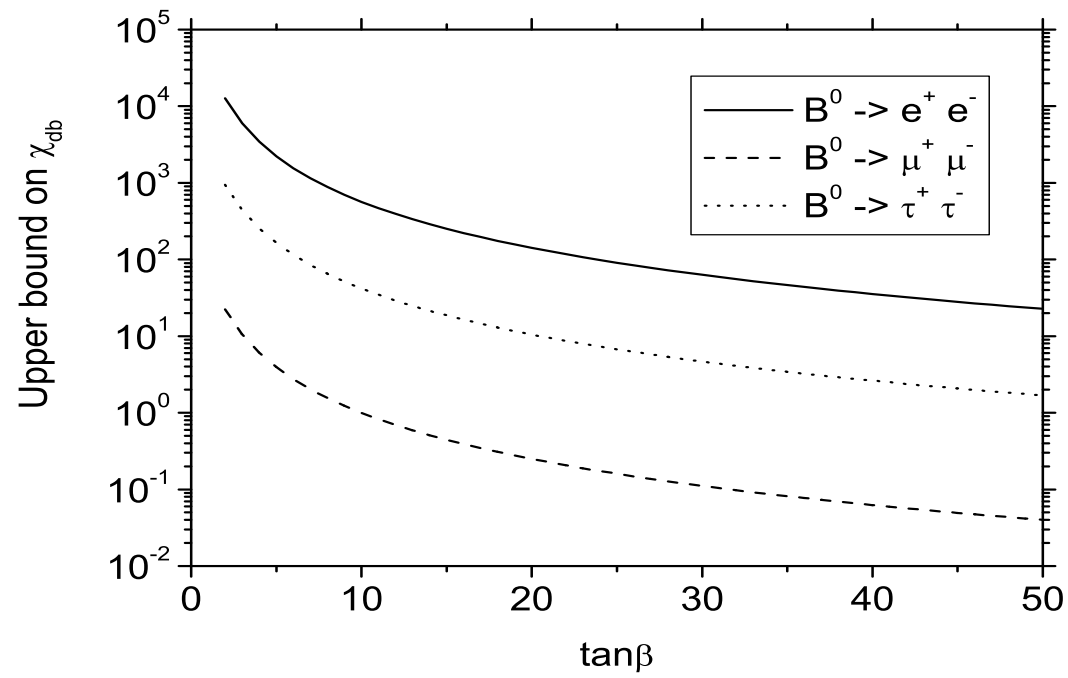

FIG. 6: Upper bound on $\chi_{d b}$ as a function of $\tan \beta$ for $m_{A^{0}}=300 \mathrm{GeV}$ (See Table III).

[4] S. Weinberg, Phys. Rev. Lett. 19 (1967) 1264; A. Salam, in Proceedings of the 8th Nobel Symposium (Stockholm), edited by N. Svartholm, (Almqvist and Wiksell, Stockholm, 1968) p. 367; S.L. Glashow, J. Illiopoulos and L. Maiani, Phys. Rev. D2 (1970) 1285

[5] URL addresses: http://www-public.slac.stanford.edu/babar, http://belle.kek.jp/

[6] A. Ilakovac and A. Pilaftsis, Nucl. Phys. B 437, 491 (1995) arXiv:hep-ph/9403398; T. Fukuyama, 


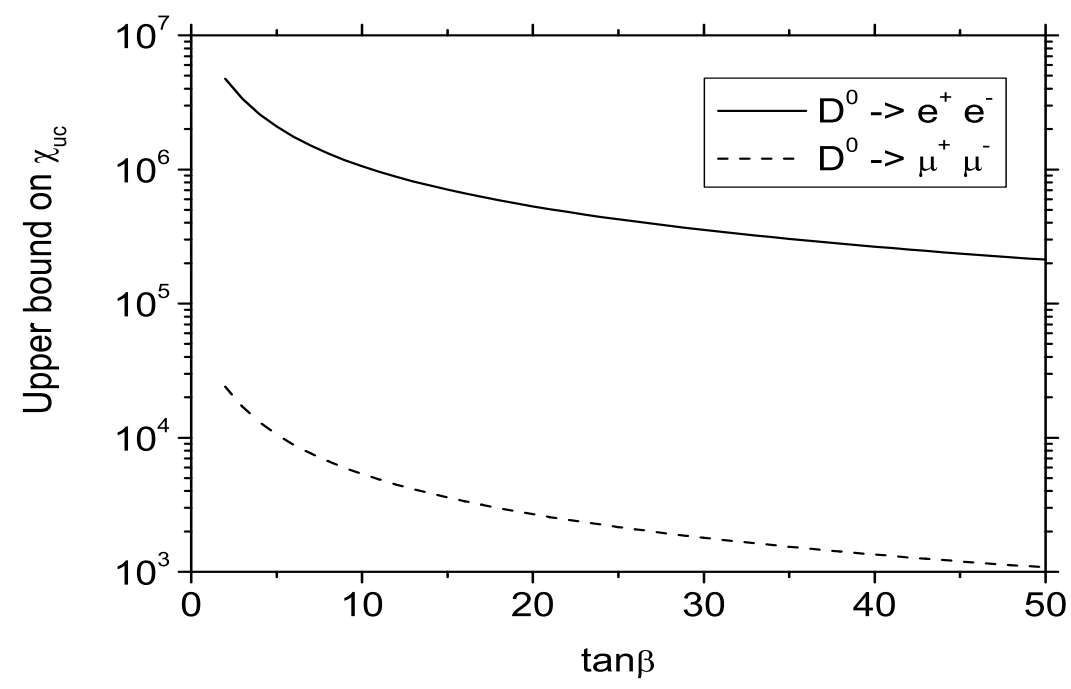

FIG. 7: Upper bound on $\chi_{u c}$ as a function of $\tan \beta$ for $m_{A^{0}}=300 \mathrm{GeV}$ (See Table III).

A. Ilakovac and T. Kikuchi, Eur. Phys. J. C 56, 125 (2008) arXiv:hep-ph/0506295; G. Cvetic, C. Dib, C. S. Kim and J. D. Kim, Phys. Rev. D 66, 034008 (2002) [Erratum-ibid. D 68, 059901 (2003)] arXiv:hep-ph/0202212; X. Y. Pham, Eur. Phys. J. C 8, 513 (1999) arXiv:hep-ph/9810484; S. Fajfer and A. Ilakovac, Phys. Rev. D 57, 4219 (1998); M. J. Herrero, J. Portoles and A. M. RodriguezSanchez, arXiv:0903.5151 [hep-ph]; E. Arganda, M. J. Herrero, J. Portoles, A. Rodriguez-Sanchez and A. M. Teixeira, AIP Conf. Proc. 1078, 335 (2009) [arXiv:0810.0163 [hep-ph]]; E. Arganda, M. J. Herrero and J. Portoles, JHEP 0806, 079 (2008) arXiv:0803.2039 [hep-ph]]; E. Arganda and M. J. Herrero, Phys. Rev. D 73, 055003 (2006) arXiv:hep-ph/0510405; S. Antusch, E. Arganda, M. J. Herrero and A. M. Teixeira, Nucl. Phys. Proc. Suppl. 169, 155 (2007) arXiv:hep-ph/0610439; Z. H. Li, Y. Li and H. X. Xu, arXiv:0901.3266 [hep-ph]; C. X. Yue, L. H. Wang and W. Ma, Phys. Rev. D 74, 115018 (2006) arXiv:hep-ph/0611054; W. j. Li, Y. d. Yang and X. d. Zhang, Phys. Rev. D 73, 073005 (2006) arXiv:hep-ph/0511273.

[7] W. Skiba and J. Kalinowski, Nucl. Phys. B 404, 3 (1993); J. L. Hewett, S. Nandi and T. G. Rizzo, Phys. Rev. D 39, 250 (1989); H. E. Logan and U. Nierste, Nucl. Phys. B 586, 39 (2000) arXiv:hep-ph/0004139; G. Lopez Castro, R. Martinez and J. H. Munoz, Phys. Rev. D 58, 033003 (1998) arXiv:hep-ph/9804368; C. S. Huang, W. Liao, Q. S. Yan and S. H. Zhu, Phys. Rev. D 63, 114021 (2001) [Erratum-ibid. D 64, 059902 (2001)] [arXiv:hep-ph/0006250; R. A. Diaz, R. Martinez and C. E. Sandoval, Eur. Phys. J. C 41, 305 (2005) arXiv:hep-ph/0406265.

[8] H. K. Dreiner, M. Kramer and B. O’Leary, Phys. Rev. D 75, 114016 (2007).

[9] V. D. Barger, J. L. Hewett and R. J. N. Phillips, Phys. Rev. D 41, 3421 (1990).

[10] W. j. Li, Y. d. Yang and X. d. Zhang, Phys. Rev. D 73, 073005 (2006) arXiv:hep-ph/0511273. 
[11] J. L. Diaz-Cruz, R. Noriega-Papaqui and A. Rosado, Phys. Rev. D 69, 095002 (2004).

[12] J. L. Diaz-Cruz, R. Noriega-Papaqui and A. Rosado, Phys. Rev. D 71, 015014 (2005).

[13] M. Gomez-Bock and R. Noriega-Papaqui, J. Phys. G 32, 761 (2006).

[14] H. Fritzsch and Z. Z. Xing, Phys. Lett. B 555, 63 (2003) (arXiv: hep-ph/0212195).

[15] For a review, see J.F. Gunion, H.E. Haber, G.L. Kane and S. Dawson, The Higgs Hunter's Guide (Addison-Wesley Publishing Company, 1990).

[16] T.P. Cheng and M. Sher, Phys. Rev. D 35 (1987) 3484.

[17] C. Amsler et al. [Particle Data Group], Phys. Lett. B 667, 1 (2008).

[18] T. Feldmann, Int. J. Mod. Phys. A 15, 159 (2000).

[19] W. M. Yao et al. [Particle Data Group], J. Phys. G 33, 1 (2006).

[20] A. Gray et al. [HPQCD Collaboration], Phys. Rev. Lett. 95, 212001 (2005).

[21] M. Wingate, C. T. H. Davies, A. Gray, G. P. Lepage and J. Shigemitsu, Phys. Rev. Lett. 92, 162001 (2004). 